\title{
Ultrasound for kidney access in percutaneous nephrolithotomy: a contemporary review
}

\author{
Alexandru Iordache', Cătălin Baston ${ }^{1,2}$, Silviu S. Guler-Margaritis ${ }^{1,2}$, Emil Angelescu ${ }^{1,2}$, \\ Vasile Cerempei ${ }^{2}$, Traxer Olivier ${ }^{3}$, Ioanel Sinescu ${ }^{1,2}$
}

${ }^{1}$ Center for Uronephrology and Renal Transplantation, Fundeni Clinical Institute, Bucharest, Romania, ${ }^{2}$ Urologic Surgery, Fundeni Clinical Institute,"Carol Davila" University of Medicine and Pharmacy, Bucharest, Romania, ${ }^{3}$ Sorbonne Universités, Université Pierre et Marie Curie, Université Paris 06, Assistance-Publique Hôpitaux de Paris, Groupe de Recherche No. 20, Groupe de Recherche Clinique sur la Lithiase Urinaire, Hôpital Tenon, Paris, France

\footnotetext{
Abstract

Nowadays percutaneous nephrolithotomy (PCNL) is the standard of care for renal staghorn calculus or large ( $>20 \mathrm{~mm})$ pelvic or caliceal stones, as well as for the failure of other treatment options.

This review aims to evaluate the contemporary use of ultrasound imaging in PCNL, by comparing it to conventional fluoroscopy, reviewing data regarding the complication and success rate of nephrostomy tract creation and stone free rate (SFR), as well as data concerning the learning curve for these procedures and cost indicators.

The evidence acquired shows that the ultrasound guided access (USGA) is a comparable method with the classic fluoroscopic guided access (FGA), with a similar safety profile, with a significant reduction in radiation exposure, up to radiation free complete PCNL. USGA PCNL seems to lead to decreased bleeding and need for transfusion, especially when the Doppler mode is used, and also to a slightly higher SFR than conventional FGA PCNL. USGA PCNL reduces the overall costs of the procedure by about $30 \%$ and can be safely learnt and performed by urologists. For an experienced endourologist, familiar with FGA PCNL, the learning curve for shifting to USGA PCNL is of approximately 20 procedures.
}

Keywords: ultrasound; fluoroscopy; percutaneous nephrolithotomy; urolithiasis

\section{Introduction}

Percutaneous nephrolithotomy (PCNL), first described by Fernström et al on three patients in 1976 [1] using radiologic guidance, is nowadays the standard of treatment for renal staghorn calculus or large $(>20 \mathrm{~mm})$ pelvic or caliceal stones, especially in the lower calyx, as well as for failure of other treatment options [2,3]. Staghorn stones seem to be the most challenging form of

Received 15.06.2018 Accepted 21.08.2018

Med Ultrason

2018, Vol. 20, No 4, 508-514

Corresponding author: Guler-Margaritis Silviu-Stelian MD, PhD, Center for Uronephrology and Renal Transplantation, Fundeni Clinical Institute, 022328, Fundeni 258, 2nd district, Bucharest, Romania;

Phone: +40212750500;

E-mail: silviu.margaritis@gmail.com urolithiasis and PCNL as monotherapy is able to achieve stone free rates (SFR) in almost $80 \%$ of cases [4].

The recent endourologic trend shows a significant increase for the ureteroscopic approach and a steady decrease for extracorporeal shockwave lithotripsy (ESWL) for renal calculi, while the percentage of stones treated by PCNL is relatively constant $[5,6]$, with a slight increase in the United States [7].

Regardless of the positioning of the patient on the operating table, taking into account that both supine and prone positions are equally safe [2], for successful PCNL, accurate puncture through the papilla of the desired calyx, in order to create the percutaneous access for stone disintegration and removal, is essential [8]. Therefore, imaging guidance is of the utmost importance in order to avoid damage to vital neighboring structures [9].

To date, real time biplanar fluoroscopy is the most frequently used technique for percutaneous puncture of 
the collecting system of the kidney, ever since it was first described by Wickham et al in 1981 [10], but the method only provides information regarding renal anatomy $[8,9,11,12]$. The main disadvantage of fluoroscopic guided access (FGA) to the kidney is that there is no real time visualization of adjacent organs, such as the bowel, liver or pleural space, with increased risk of injury to these structures $[13,14]$. Also, this approach exposes the patient and the surgical staff to radiation, which is lower but significant for the surgeon [15-17] . The patient is exposed to a mean of $8.66 \mathrm{mSv}$ [18] during a PCNL session, with a peak in the first step, when gaining access to the collecting system [19], the risk of irradiation being dose dependent [20]. Mancini et al [18] revealed that patients with increased BMI, complex stone burden and multiple access tracts are at an increased risk of irradiation during PCNL, from which the most exposed are the obese patients [21].

The use of ultrasonography for access (USGA) in PCNL was first described in the 1970s, with increasing popularity and growing literature data demonstrating its advantages in terms of efficacy, safety and feasibility for upper urinary tract lithiasis [22]. The first ultrasound guided PCNL was reported almost 20 years ago by Desai et al [23] on a pediatric patients series. The advantages of ultrasound as an imaging modality are obvious, in terms of real time imaging, with proper anatomic description, without ionizing radiation, cheaper to use and readily available, with application in virtually any interventional procedure, with a reduced risk of complications as stated by the World Federation for Ultrasound in Medicine and Biology (WFUMB) in 2015 [24]. These advantages also apply for imaging guidance for PCNL access, with proven efficacy in pregnancy [13], with drastic reduction of radiation exposure for the obese patients [21]. The main disadvantage is the difficulty to puncture non-dilated collecting system, as well as poor visualization of the guidewire and sometimes low visualization of the punctureneedle $[25,26]$. To our knowledge, to date there is no consensus on the optimal access to the collecting system for PCNL and no recommendation provided by either the European Association of Urology or the American Association of Urology guidelines regarding percutaneous access to the kidney, with only a small amount of data comparing USGA and FGA in PCNL published in the last five years.

The purpose of this review is to assess the contemporary use of ultrasound imaging in PCNL, in comparison with conventional fluoroscopic guidance, reviewing data concerning ultrasound as an imaging method in PCNL and accounting for complications, success rate of nephrostomy tract creation and SFR.

\section{Methods}

In March 2018, we performed a MEDLINE/PubMed search for papers published in English, in peer-reviewed journals in the last 15 years, using the following keywords: PCNL, ultrasound, fluoroscopy, percutaneous, access, nephrolithotomy and urolithiasis. Out of 48 papers selected and reviewed after excluding paediatric stone disease, we refined the search on papers regarding the comparison between ultrasound guided and classic fluoroscopic guided access to the kidney for PCNL. We selected six papers that we found to be most relevant and which met modern study criteria (Table I). Taking into account or analyzing the mean stone size, access time, operating time, fluoroscopy time for accessing the kidney, success rate of puncture, complications, SFR and the need for further additional procedures for improving $S F R$, as well as mean hospital stay. We also reviewed data concerning the learning curve for USGA PCNL and the impact on costs of the procedure when switching to ultrasound guided access, which is of great importance, especially in high volume centers.

\section{Results}

Data regarding the average stone size for PCNL approach showed a maximum diameter above $20 \mathrm{~mm}$ in most of the papers. Mean access time was roughly similar, with the exception of the trial conducted by Basiri et al [27], which found that FGA was performed two times faster than USGA, and Agarwal et al [28], who reported quite the opposite, without any explanation. In both studies the procedure was performed by the urologist, in a prone position, targeting the inferior posterior calyx. This might be explained by the surgeon's experience and familiarity with USGA. Total operating time was similar between USGA and FGA, as well as the puncture success rate. Fluoroscopy time was significantly lower for the USGA groups. With the exception of Basiri et al [27], where the SFR was not one of the outcomes and was not reported, all the other studies revealed similar or higher SFR for the USGA approach. Complications were similar, but a significant decrease in bleeding and need for transfusion for the USGA arms was reported in two studies $[29,30]$. Length of hospital stay data was contradictory, Andonian et al [29] reporting a mean of 5.3 days for USGA PCNL versus 3.5 days for FGA and $\mathrm{Ng}$ et al [22] 2.9 days for the USGA group versus 4.6 days for the FGA group. The results of $\mathrm{Ng}$ et al [22] do not correlate with the SFR or with complication or retreatment rate. On the other hand, Wang et al [30] found no significant difference in hospital stay between the two groups in their me- 


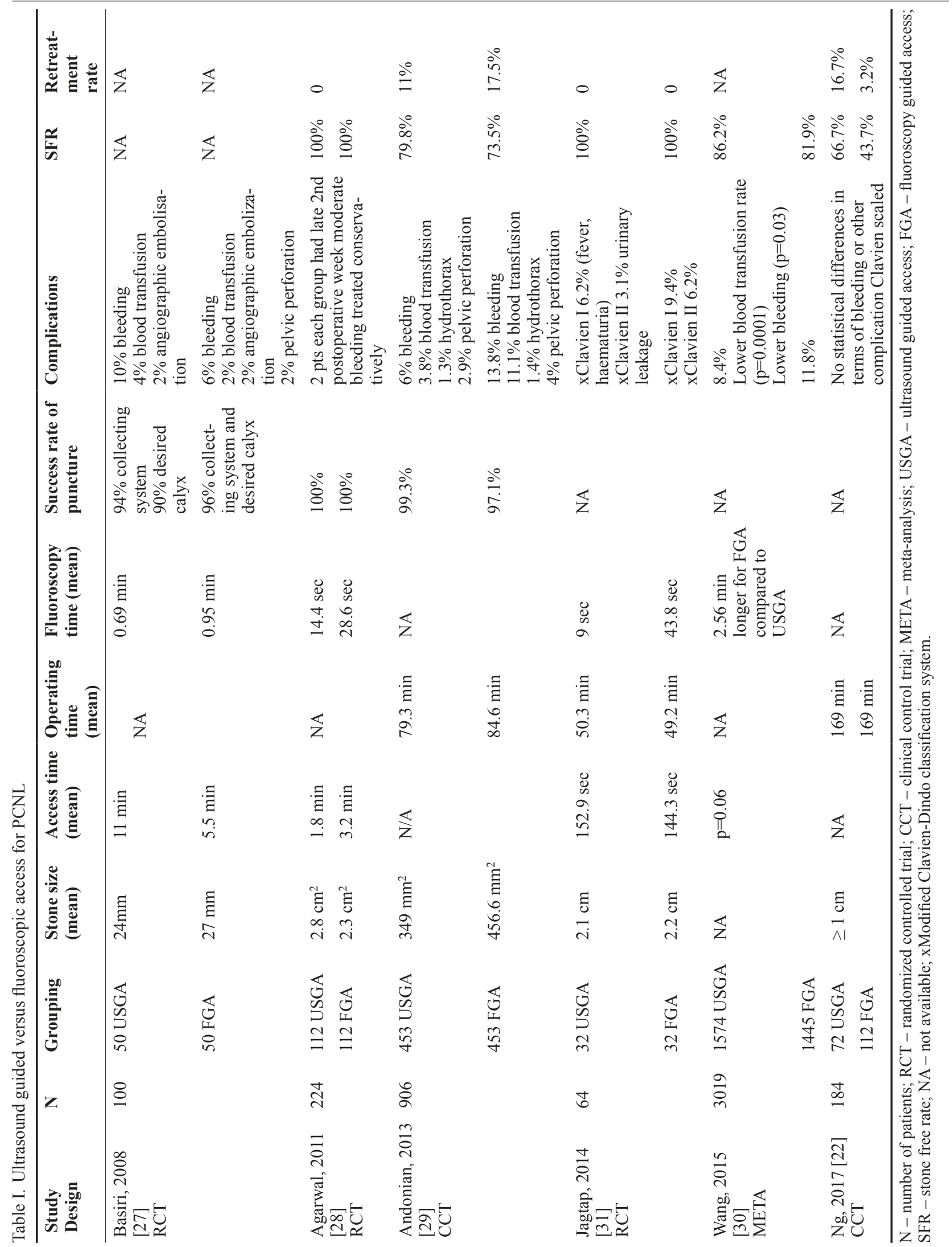


ta-analysis comprising over 3000 patients. Out of the comparison between USGA and FGA for PCNL some important aspects emerged, such as the significant reduction in radiation exposure for both the patient and the surgical staff, which was somehow predictable, mainly due to the harmless principle of ultrasound, with similar puncture time and total operative time. However, fluoroscopy was necessary as an adjunct for verifying correct needle placement or in case of failed USGA, as well as in conjunction to ultrasound for tract creation after initial puncture $[27,28,31]$. Data on mean access time are heterogenous, with the exception of Wang et al [30] who found no statistically significant difference between the groups on their meta-analysis on 3019 patients enrolled in 14 studies. Puncture success rate are similar, with generally mild complications of grade I and II according to the Clavien-Dindo scale [32]. However, the operative bleeding and subsequent need for transfusion seem to be lower for the USGA groups $[29,30]$, which is probably due to the higher number of punctures necessary to gain access, and perhaps to some extent to the increased operative time of FGA PCNL. These findings are sustained by the results of CROES (The Clinical Research Office of the Endourological Society) PCNL Global study [33], that showed a significantly higher access rate failure for FGA compared with USGA (2.9 vs. $0.7 \%$ ) in their complex analysis of almost 6000 patients. The most important parameter of the outcome of PCNL is the SFR, which seems to be higher in the USGA PCNL groups, owing to the superiority of ultrasound in depicting renal anatomy and the ability of identifying radiolucent stones $[22,29,30]$.

Another advantage of USGA is the use of Doppler mode, which can depict renal vasculature and can facilitate needle puncture without injury to significant vessels that can lead to severe bleeding. Tzeng et al [34] evaluated the use of Doppler mode in USGA PCNL, in a prospective randomized controlled trial comparing USGA in standard B-mode with Doppler mode. The study revealed a significantly lower bleeding and subsequent need for transfusion in the group where Doppler mode was used. $\mathrm{Li}$ et al [35] recently published their experience over 9 years including 8025 patients with urolithiasis that were subjected to PCNL with access and tract dilation performed solely under Doppler ultrasound guidance. They reported a mean stone size of $32 \mathrm{~mm}$, with an operating time of 42 min (mean). SFR achieved was $85.5 \%$, with a mean hospital stay of 4.1 days. Almost $5 \%$ of patients complicated with fever $>38.5^{\circ} \mathrm{C}$, while the need for transfusion resumed at only $1.1 \%$. Pleural injury was reported in 15 cases $(0.19 \%)$. Only four patients $(0.05 \%)$ needed conversion to open surgery, out of which two patients re- quired a nephrectomy. These results sustain once more the safety and feasibility of the Doppler mode in USGA.

Last but not least, several papers reported that the puncture of the collecting system, either by USGA or FGA, was performed entirely by the urologist $[27,28]$. This should encourage clinicians to perform USGA with fluoroscopic guidance as a complementary imaging technique, with the same safety profile and SFR of both methods, but with significant reduction of the radiation exposure. Data from the CROES study [33] revealed that the access was obtained in $90 \%$ of cases by the urologist. Several papers reported a comparison between the access obtained by the radiologist versus the urologist, with roughly similar complication rates but slightly higher SFR for the cases where the access was obtained by the surgeon [36-38]. Some authors even recommend that the urologist and not the interventional radiologist should perform the renal puncture under ultrasound guidance, for optimal access and avoidance of collateral organ injuries [39].

Furthermore, in a recent study Hudnall et al [40] analyzed 71 consecutive PCNL performed using either USGA or FGA finding a mean reduction of overall costs of $30 \%$ when using USGA, which makes the ultrasound approach even more appealing.

The main disadvantage of USGA is the puncture of the non-dilated collecting system [9], which was overcome by placing an uretheral catheter and injecting saline solution, sometimes with some contrast agent [22,28,31], or even a small amount of atmospheric air, for better visualization of the caliceal anatomy [27]. Moreover, the ureteral catheter prevents the migration of stone fragments to the ureter through the PCNL procedure. Agarwal et al [28] administered a $10 \mathrm{mg}$ IV bolus of furosemide to all 224 patients in their study, for uniform and sustained renal collecting system dilatation throughout the procedure. This simple artifice acts as a great aid, but there is still the disadvantage of repositioning the patient from lithotomy position to the desired position for PCNL, most often under general anesthesia, which increases operating time and can produce cervical spine injury, ocular or peripheral nerves lesions, as well as ventilatory and cardiac alterations, especially when the preffered position is prone [41]. This is an important aspect, as in the CROES global study [33], PCNL was performed in the prone position in $80 \%$ of cases of their large cohort study.

However, in the attempt of further compensation of this disadvantage, Usawachintachit et al [42] assessed the feasibility of using contrast enhanced ultrasound (CEUS), by administering a small amount of ultrasonographic contrast (Optison ${ }^{\mathrm{TM}}$, GE Healthcare) through a retrograde placed ureteral catheter, on a pilot series of 
five patients with urolithiasis with indication for PCNL, but with non-dilated renal units. Puncture was successful in all cases, without side-effects related to the ultrasound contrast. Future research is warranted to validate this method of aiding renal access in non-dilated kidneys.

Finally, we reviewed data concerning the learning curve for both FGA and USGA. Allen et al [43] published in 2005 their experience studying the learning curve of an inexperienced urologist in performing FGA PCNL, under the supervision of an highly trained endourologist. They found that the operating time decreased from a mean of $142 \mathrm{~min}$ for the first 15 cases to $92 \mathrm{~min}$ for cases 46-60, where it stabilized for the next cases, up to 155 cases. The results were comparable with the timing of the experienced surgeon (mean of $98 \mathrm{~min} /$ procedure) after the first 60 operations. Fluoroscopy time and radiation dose decreased with the gain in surgical experience, reaching a plateau after 115 procedures, which was again similar with the results of the expert urologist. Therefore, the authors concluded that a mean of 60 procedures are required for achieving a good level of competence, excellence being achievable after 115 procedures. Similar results were reported by Tanriverdi et al [44], who found that approximately 60 procedures are necessary for a non-experienced urologist to gain competence in FGA PCNL, as after this amount there was no significant drop in operating time or radiation exposure. More recent data suggest that at least 50 procedures of FGA PCNL are necessary in order to achieve reliable skills in accessing the collecting system [45].

On the other hand, Song et al [46] studied the learning curve for USGA PCNL, on a series of 120 cases in which USGA PCNL was performed by an unexperienced surgeon with some endourologic background and a month of observing and assisting over 20 USGA PCNL procedures. The results were compared to those of an expert endourologist with over 1000 PCNL's. They found that the novice reached comparable operating time, ultrasound screening time and tract dilation comparable to those of the expert urologist after 60 procedures. The complication rate decreased with experience gained, but without statistical significance. There were only Clavien I and II grade complications. The SFR increased with experience and achieved an overall of $75 \%$ for all the procedures. Similar findings were reported by Yu et al [47], their study concluded that 60 procedures are necessary for proper USGA PCNL experience, 120 represent excellent performance and nearly perfect results are obtained after 180 procedures.

A recent paper published by Usawachintahit et al [48] studied the learning curve necessary for an experienced endourologist with training in FGA PCNL to ob- tain proper skills for USGA PCNL. At first, the surgeon performed only the access to the kidney with the aid of ultrasound, then switching to fluoroscopic imaging. During the study he gradually performed further steps with the aid of ultrasound, achieving almost X-ray free USGA PCNL at the end of the study. Fluoroscopy was also used for failed USGA attempts. Fluoroscopy time decreased nearly sevenfold between the first 20 cases and the last 20, while radiation dose decreased over sevenfold in the same manner. Puncture success rate was about $30 \%$ in the first 20 cases then rising to $75 \%$ from the $21^{\text {st }}$ case, reaching $100 \%$ at the end of the study. Complications were mild, Clavien I and II group, comparable with the fluoroscopy group. Overall SFR reached $88 \%$. They concluded that after approximately 20 procedures, USGA PCNL can be performed safely and effective by a previously experienced FGA PCNL urologist. These findings are important because they reflect a relative learning curve not for a novice surgeon in the field of PCNL, but for those already experienced that want to shift to a less irradiating method, without sacrificing the surgical outcome.

\section{Conclusions}

The evidence acquired shows that the ultrasound guided access is a comparable method with the classic fluoroscopic guided access, with a similar safety profile and complication rate and a significant reduction in radiation exposure for both patient and surgeon and up to radiation free complete PCNL, although fluoroscopic guided access to the kidney remains the most popular access path to the kidney for PCNL. However, USGA PCNL seems to lead to decreased bleeding and a need for a transfusion, especially when the Doppler mode is used, and also to a slightly higher SFR than conventional FGA PCNL. The two methods have similar hospital stay, but it seems that USGA PCNL reduces the overall costs of the procedures by about $30 \%$. USGA PCNL can be safely performed by urologists, therefore they should be encouraged to learn and apply this method. For the experienced endourologist familiar with FGA PCNL, the learning curve for shifting to USGA PCNL is of approximately 20 procedures. We consider that more prospective clinical trials are required in order to obtain consensus. Also, further research of simple tricks such as the injection of ultrasonographic contrast or air in the collecting system to increase the visualization of the kidney anatomy is warranted for reducing the difficulty of USGA PCNL.

Conflict of interest: none 


\section{References}

1. Fernström I, Johansson B. Percutaneous pyelolithotomy. A new extraction technique. Scand. J Urol Nephrol 1976; 10:257-259.

2. Türk C, Neisius A, Petrik A, et al. EAU Guidelines on Urolithiasis. Limited update March 2017. European Association of Urology 2017. Available at: https://uroweb.org/wp-content/uploads/EAU-Guidelines-on-Urolithiasis 2017 1005V2.pdf

3. Assimos D, Krambeck A, Miller NL, et al. Surgical Management of Stones: American Urological Association/Endourological Society Guideline, PART II. J Urol 2016;196:1161-1169.

4. Preminger GM, Assimos DG, Lingerman JE, et al. Chapter 1: AUA guideline on management of staghorn calculi: diagnosis and treatment recommendations. J Urol 2005; 173:1991-2000.

5. Oberlin DT, Flum AS, Bachrach L, Matulewicz LS, Flury SC. Contemporary surgical trends in the management of upper tract calculi. J Urol 2015;193:880-884.

6. Doizi S, Raynal G, Traxer O. Évolution du traitement chirurgical de la lithiase urinaire sur 30 ans dans un centre hospitalo-universitaire. Prog Urol 2015;25:543-548.

7. Morris DS, Wei JT, Taub DA, Dunn RL, Wolf JS Jr, Hollenbeck BK. Temporal trends in the use of percutaneous nephrolithotomy. J Urol 2006;175:1731-1736.

8. Miller NL, Matlaga BR, Lingeman JE. Techniques for Fluoroscopic Percutaneous Renal Access. J Urol 2007; 178:15-23.

9. Kalogeropoulou C, Kallidonis P, Liatsikos EN. Imaging in percutaneous nephrolithotomy. J Endourol 2009;23:15711577.

10. Wickham JE, Kellett MJ. Percutaneous nephrolithotomy. Br J Urol 1981;53:297-299.

11. Steinberg PL, Semins MJ, Wason SEL, Matlaga BR, Pais VM. Fluoroscopy-guided percutaneous renal access. J Endourol 2009;23:1627-1631.

12. Park S, Pearle MS. Imaging for Percutaneous Renal Access and Management of Renal Calculi. Urol Clin North Am 2006;33:353-364.

13. Ganpule AP, Vijayakumar M, Malpani A, Desai MR. Percutaneous nephrolithotomy (PCNL) a critical review. Int J Surg 2016;36:660-664.

14. Tzou DT, Usawachintachit M, Taguchi K, Chi T. Ultrasound Use in Urinary Stones: Adapting Old Technology for a Modern-Day Disease. J Endourol 2017;31(S1):S89-S94.

15. Galonnier F, Traxer O, Rosec M, et al. Surgical Staff Radiation Protection During Fluoroscopy-Guided Urologic Interventions. J Endourol 2016;30:638-643.

16. Ritter M, Schmitt M, Siegel F, et al. 704 The DEFI-Study - Dosimetry in endourologic fluoroscopic interventions risk of radiation exposure for endourologic surgeons. Eur Urol Suppl 2011;10:225.

17. Wenzler DL, Abbott JE, Su JJ, et al. Predictors of radiation exposure to providers during percutaneous nephrolithotomy. Urol Ann 2017;9:55-60.
18. Mancini JG, Raymundo EM, Lipkin M, et al. Factors Affecting Patient Radiation Exposure During Percutaneous Nephrolithotomy. J Urol 2010;184:2373-2377.

19. Demirci A, Raif Karabacak O,Yalçınkaya F, Yiğitbaşı O, Aktaş C. Radiation exposure of patient and surgeon in minimally invasive kidney stone surgery. Prog Urol 2016;26:353-359.

20. Safak M, Olgar T, Bor D, Berkmen G, Gogus C. Radiation doses of patients and urologists during percutaneous nephrolithotomy. J Radiol Prot 2009;29:409-415.

21. Usawachintachit M, Masic S, Chang HC, Allen IE, Chi T. Ultrasound Guidance to Assist Percutaneous Nephrolithotomy Reduces Radiation Exposure in Obese Patients. Urology 2016;98:32-38.

22. Ng FC, Yam WL, Lim TYB, Teo JK, Ng KK, Lim SK. Ultrasound-guided percutaneous nephrolithotomy: Advantages and limitations. Investig Clin Urol 2017;58:346-352.

23. Desai M, Ridhorkar V, Patel S, Bapat S, Desai M. Pediatric percutaneous nephrolithotomy: assessing impact of technical innovations on safety and efficacy. J Endourol 1999; 13:359-364.

24. Dietrich CF, Goudie A, Chiorean L, et al. Point of Care U1trasound: A WFUMB Position Paper. Ultrasound Med Biol 2017;43:49-58.

25. Lojanapiwat B. The ideal puncture approach for PCNL: Fluoroscopy, ultrasound or endoscopy? Indian J Urol 2013;29:208-213.

26. Chu C, Masic S, Usawachintachit M, et al. UltrasoundGuided Renal Access for Percutaneous Nephrolithotomy: A Description of Three Novel Ultrasound-Guided Needle Techniques. J Endourol 2016;30:153-158.

27. Basiri A, Ziaee AM, Kianian HR, Mehrabi S, Karami H, Moghaddam SM. Ultrasonographic versus Fluoroscopic Access for Percutaneous Nephrolithotomy: A Randomized Clinical Trial. J Endourol 2008;22:281-284.

28. Agarwal M, Agrawal MS, Jaiswal A, Kumar D, Yadav H, Lavania P. Safety and efficacy of ultrasonography as an adjunct to fluoroscopy for renal access in percutaneous nephrolithotomy (PCNL). BJU Int 2011;108:1346-1349.

29. Andonian S, Scoffone CM, Louie MK, et al. Does Imaging modality used for percutaneous renal access make a difference? A matched case analysis. J Endourol 2013;27:2428.

30. Wang K, Zhang P, Xu X, Fan M. Ultrasonographic versus Fluoroscopic Access for Percutaneous Nephrolithotomy: A Meta-Analysis. Urol Int 2015;95:15-25.

31. Jagtap J, Mishra S, Bhattu A, Ganpule A, Sabnis R, Desai MR. Which Is the preferred modality of renal access for a trainee urologist: ultrasonography or fluoroscopy? Results of a prospective randomized trial. J Endourol 2014;28:1464-1469.

32. Clavien PA, Barkun J, de Oliveira ML, et al. The claviendindo classification of surgical complications: Five-year experience. Ann Surg 2009;250:187-196.

33. Kamphuis GM, Baard J, Westendarp M, de la Rosette JJ. Lessons learned from the CROES percutaneous nephrolithotomy global study. World J Urol 2015;33:223-233. 
34. Tzeng BC, Wang CJ, Huang SW, Chang CH. Doppler Ultrasound-guided Percutaneous Nephrolithotomy: A Prospective Randomized Study. Urology 2011;78:535-539.

35. Li J, Xiao B, Hu W, Yang B, Chen L, Hu H, Wang X. Complication and safety of ultrasound guided percutaneous nephrolithotomy in 8,025 cases in China. Chin Med J (Engl) 2014;127:4184-4189.

36. Ingimarsson J, Bechis S, Moses R, Johnson E, Eisner B, Pais V. MP27-16 Comparsion of access related complications: radiologist vs urologist obtained percutaneous renal access. J Urol 2014;191:e280.

37. Wang Y, Lu Z, Hu J, et al. Renal access by sonographer versus urologist during percutaneous nephrolithotomy. Urol J 2014;10:1035-1039.

38. Watterson JD, Soon S, Jana K. Access related complications during percutaneous nephrolithotomy: urology versus radiology at a single academic institution. J Urol 2006;176:142-145.

39. Osman M, Wendt-Nordahl G, Heger K, Michel MS, Alken $\mathrm{P}$, Knoll T. Percutaneous nephrolithotomy with ultrasonography-guided renal access: Experience from over 300 cases. BJU Int 2005;96:875-878.

40. Hudnall M, Usawachintachit M, Metzler I, et al. Ultrasound Guidance Reduces Percutaneous Nephrolithotomy Cost Compared to Fluoroscopy. Urology 2017;103:52-58.

41. Zhao Z, Fan J, Liu Y, de la Rosette J, Zeng G. Percutaneous nephrolithotomy: position, position, position! Urolithiasis 2018;46:79-86.
42. Usawachintachit M, Tzou DT, Mongan J, Taguchi K, Weinstein S, Chi T. Feasibility of Retrograde Ureteral Contrast Injection to Guide Ultrasonographic Percutaneous Renal Access in the Nondilated Collecting System. J Endourol 2017;31:129-134.

43. Allen D, O'Brien T, Tiptaft R, Glass J. Defining the learning curve for percutaneous nephrolithotomy. J Endourol 2005; 19:279-282.

44. Tanriverdi O, Boylu U, Kendirci M, Kadihasanoglu M, Horasanli K, Miroglu C. The Learning Curve in the Training of Percutaneous Nephrolithotomy. Eur Urol 2007;52:206211.

45. Negrete-Pulido O, Molina-Torres M, Castaño-Tostado E, Loske AM, Gutiérrez-Aceves J. Percutaneous renal access: the learning curve of a simplified approach. J Endourol 2010;24:457-460.

46. Song Y, Ma Y, Song Y, Fei X. Evaluating the Learning Curve for Percutaneous Nephrolithotomy under Total Ultrasound Guidance. PLoS One 2015; 10:e0132986.

47. Yu W, Rao T, Li X, et al. The learning curve for access creation in solo ultrasonography-guided percutaneous nephrolithotomy and the associated skills. Int Urol Nephrol 2017;49:419-424.

48. Usawachintachit M, Masic S, Allen IE, Li J, Chi T. Adopting Ultrasound Guidance for Prone Percutaneous Nephrolithotomy: Evaluating the Learning Curve for the Experienced Surgeon. J Endourol 2016;30:856-863. 